Accepted to the Astrophysical Journal March 10, 2013

Preprint typeset using IATEX style emulateapj v. 5/2/11

\title{
A SAMPLE OF OB STARS THAT FORMED IN THE FIELD
}

\author{
M. S. Oey, J. B. Lamb, C. T. Kushner, E. W. Pellegrini ${ }^{1}$, and A. S. Graus ${ }^{2}$ \\ Department of Astronomy, University of Michigan, 830 Dennison Building, 500 Church Street, Ann Arbor, MI, 48109-1042 \\ Accepted to the Astrophysical Journal March 10, 2013
}

\begin{abstract}
We present a sample of $14 \mathrm{OB}$ stars in the Small Magellanic Cloud that meet strong criteria for having formed under extremely sparse star-forming conditions in the field. These stars are a minimum of $28 \mathrm{pc}$ in projection from other OB stars, and they are centered within symmetric, round HiI regions. They show no evidence of bow shocks, implying that the targets are not transverse runaway stars. Their radial velocities relative to local Hi also indicate that they are not line-of-sight runaway stars. A friends-of-friends analysis shows that 9 of the objects present a few low-mass companion stars, with typical mass ratios for the two highest-mass stars of around 0.1. This further substantiates that these OB stars formed in place, and that they can and do form in extremely sparse conditions. This poses strong constraints on theories of star formation and challenges proposed relations between cluster mass and maximum stellar mass.

Subject headings: stars: formation — stars: luminosity function, mass function — stars: massive - open clusters and associations: general — galaxies: star clusters: general galaxies: stellar content — Magellanic Clouds
\end{abstract}

\section{INTRODUCTION}

One of the most important constraints on theories of massive star formation is the degree to which massive, $\mathrm{OB}$ stars can form in true isolation (see discussion in, e.g., Lamb et al. 2010). Our conventional understanding of the stellar initial mass function (IMF) dictates that a large population of lower-mass stars should generally accompany the formation of massive stars. Similarly, the initial cluster mass function can be described as a clustering law where the number of massive stars $N_{*}$ is described by an inverse power-law function almost identical to the IMF (e.g., Oey 2011; Oey \& Clarke 1998; Elmegreen \& Efremov 1997). Both the IMF and initial cluster mass function behave as probability density functions, so if they are invariant, it implies that individual OB stars should form in isolation on rare occasions. But does Nature actually operate that way?

Isolated field $\mathrm{OB}$ stars are certainly known to exist. However, whether any truly isolated OB stars actually formed in situ is presently controversial. A substantial fraction of the field massive star population is known to consist of runaway stars ejected from clusters but the contribution, if any, of an in-situ component is not established. However, several lines of evidence support the formation of $\mathrm{O}$ stars in extremely sparse groups, if not in isolation (Oey \& Lamb 2012): (1) The number of field $\mathrm{O}$ stars defined by a friends-of-friends algorithm falls smoothly onto the power-law $N_{*}$ distribution for groups and clusters (Oey et al. 2004). This implies that the field star population simply represents the low-mass extreme for the mass spectrum of clustered star formation. While thus far this has been evaluated only for the Small Magellanic Cloud (SMC), the star formation properties in this galaxy are unremarkable, and the relation most likely

\footnotetext{
${ }^{1}$ Present address: Department of Physics and Astronomy, University of Toledo, $2801 \mathrm{~W}$. Bancroft, Toledo, OH 43606

${ }^{2}$ Present address: Department of Physics and Astronomy, University of California, Irvine, CA 92697
}

applies in other star-forming galaxies as well. (2) The IMF for massive field stars is substantially steeper than for clusters and associations in the Magellanic Clouds (Lamb et al. 2013; Massey 2002; Massey et al. 1995), having a power-law slope $\Gamma \lesssim-2.3$ in contrast to the Salpeter (1955) value of -1.35 . This is perhaps the only systematic variation in the IMF that is now well established. Since the frequency of runaway stars increases with mass (e.g., Moffat 1998; Gies 1987), which would flatten the inferred IMF, the steep field IMF suggests a major, if not dominant, contribution of in situ stars. (3) The distribution of rotation velocities for field earlytype stars is also well established to differ from that for cluster members (e.g., Guthrie 1984; Wolff et al. 2007), with the field $v \sin i$ strongly weighted to low values relative to clusters. This may be an evolutionary spin-down effect (Huang \& Gies 2008), or it may be intrinsic to the star-formation density (Wolff et al. 2007). (4) Last, but not least, there are numerous examples of candidate in situ massive field stars. Lamb et al. (2010) and de Wit et al. $(2004,2005)$ identify OB stars with sparse associated groups of lower-mass stars, suggesting that a significant fraction of massive stars form in such extreme, low-density environments. Similarly, Testi et al. (1997, 1999) identify isolated Herbig Ae/Be stars. In that case in particular, it is difficult to reconcile the isolation of these stars with their youth.

Together, these arguments strongly suggest that massive stars occasionally do form in relative isolation. It was already suggested decades ago that high-mass stars may sometimes form in the lower-density cluster outskirts (Burki 1978). However, since clusters may disperse quickly, especially when a single massive star is present (e.g., Allison et al. 2009), it has also been suggested that essentially all massive field stars are runaways from clusters (e.g., Gvaramadze et al. 2012). The strongest evidence for the in-situ formation of field massive stars is to identify newborn, isolated massive stars. The Herbig Ae/Be stars of Testi et al. are an important such 
Table 1

SMC in-situ field OB stars

\begin{tabular}{|c|c|c|c|c|c|c|c|c|c|c|c|c|}
\hline Star $^{\mathrm{a}}$ & Sp Type ${ }^{b}$ & RA and $\mathrm{D}$ & ec $(J 2000)^{c}$ & $\mathrm{RV}^{\mathrm{d}}$ & $v(\mathrm{HI})^{\mathrm{d}}$ & $V^{\mathrm{c}}$ & $A_{V}{ }^{\mathrm{e}}$ & $l(\mathrm{pc})$ & $N($ tot $)$ & $N(\mathrm{MS})$ & $m_{1}\left(M_{\odot}\right)^{\mathrm{b}}$ & $m_{2} / m_{1}$ \\
\hline 1600 & O8.5 V & 0:42:09.92 & $-73: 13: 56.9$ & 93 & 103 & 14.725 & 0.42 & 0.98 & 3 & 2 & 23.6 & 0.11 \\
\hline 3173 & $\mathrm{O}+$ neb & $0: 43: 36.69$ & $-73: 02: 26.9$ & 111 & 111 & 15.529 & 0.37 & 1.06 & 5 & 3 & $25-40$ & $\leq 0.2$ \\
\hline 17813 & B0 V & 0:50:49.99 & $-73: 24: 22.3$ & 192 & 184 & 15.101 & 0.38 & 0.91 & 0 & 0 & 18.3 & \\
\hline 24119 & $\mathrm{~B} 1-2.5 \mathrm{~V}$ & $0: 52: 38.19$ & $-73: 26: 17.1$ & 170 & 170 & 15.272 & 0.27 & 0.91 & 4 & 3 & 14.8 & 0.16 \\
\hline 35491 & O8 V & $0: 55: 59.59$ & $-72: 19: 54.4$ & 126 & 126 & 14.856 & 0.47 & 0.83 & 0 & 0 & 30.4 & $\ldots$ \\
\hline 36514 & O9 V & $0: 56: 17.33$ & $-72: 17: 28.8$ & 141 & 141 & 15.385 & 0.33 & 0.98 & 12 & 7 & 20.5 & 0.12 \\
\hline 64453 & B1 Ib & $1: 06: 40.30$ & $-73: 10: 24.6$ & 194 & 194 & 13.506 & 0.29 & 1.59 & 2 & 2 & 19.0 & 0.12 \\
\hline 66415 & O9.7 Ia & $1: 07: 40.40$ & $-72: 51: 00.0$ & 185 & 185 & 13.285 & 0.26 & 1.44 & 4 & 2 & 34.5 & 0.08 \\
\hline 67334 & B0 V & 1:08:08.03 & $-72: 38: 19.7$ & 173 & 173 & 15.270 & 0.43 & 1.44 & 4 & 1 & 16.5 & 0.19 \\
\hline 69598 & O9 V & $1: 09: 26.78$ & $-72: 01: 26.9$ & 164 & 168 & 15.640 & 0.23 & 2.04 & 11 & 6 & 17.6 & 0.51 \\
\hline 70149 & $09.5 \mathrm{~V}$ & $1: 09: 48.26$ & $-72: 30: 19.4$ & 117 & 126 & 14.755 & 0.31 & 1.51 & 3 & 0 & 23.8 & $\ldots$ \\
\hline 71409 & $\mathrm{Be}$ & $1: 10: 45.17$ & $-72: 21: 37.5$ & 184 & 184 & 15.029 & 0.25 & 1.82 & 0 & 0 & $15-20$ & $\cdots$ \\
\hline 71815 & $\mathrm{O} 8 \mathrm{~V}$ & $1: 11: 05.63$ & $-72: 13: 41.7$ & 172 & 172 & 15.495 & 0.18 & 1.97 & 0 & 0 & 19.1 & $\ldots$ \\
\hline 75984 & B0.2 V & $1: 15: 14.70$ & $-72: 20: 19.4$ & 216 & $213^{f}$ & 15.693 & 0.31 & 2.49 & 11 & 7 & 14.0 & 0.36 \\
\hline
\end{tabular}

a From Massey (2002).

b From Lamb et al. (2013).

c From OGLE III (Udalski et al. 2008).

d Velocity in $\mathrm{km} \mathrm{s}^{-1}$ of the HI component having brightness temperature $\geq 20 \mathrm{~K}$, that is nearest to the stellar RV.

e From Zaritsky et al. (2002).

${ }^{\mathrm{f}} \mathrm{HI}$ is only observed for velocities $<215 \mathrm{~km} \mathrm{~s}^{-1}$, so this value is most likely a lower limit.

data set. More recently, Selier et al. (2011) identify the SMC object N33 as an isolated, compact HiI region hosting a candidate isolated field $\mathrm{O}$ star, and Bressert et al. (2012) present a candidate sample of relatively isolated O stars near the 30 Doradus giant star-forming complex. We also note a number of spherical, Strömgren sphere HiI regions with centrally positioned stars identified by Zastrow et al. (2013) in the Large Magellanic Cloud. In this contribution, we present a substantial sample of 14 field OB stars in the SMC hosting single-star HII regions that appear to imply the in-situ formation of these stars.

\section{SMC FIELD STARS IN STRÖMGREN SPHERES}

Oey et al. (2004) determined the SMC clustering law for massive stars by applying a friends-of-friends algorithm to photometrically identified $\mathrm{OB}$ star candidates. Any stars not within the 28-pc clustering length from other candidates are defined to be field stars, thereby yielding an essentially complete sample of field stars in this galaxy. We carried out a complete spectroscopic survey of this field star sample, the Runaways and Isolated O-Type Star Spectroscopic Survey of the SMC (RIOTS4), using the IMACS imaging spectrograph at Magellan with multislit masks (Lamb et al. 2013; Oey \& Lamb 2012). This survey yields, among other things, spectral types and radial velocities of the target stars. Critical insight on the origin of these stars can be provided by the HiI regions that they generate, which can constrain the likelihood that they formed in the observed locations. Fortuitously, we recently completed a new catalog of HiI regions in the Magellanic Clouds (Pellegrini et al. 2012, 2013) using the $\mathrm{H} \alpha$, [OIII], and [SII] narrow-band imaging data from the Magellanic Clouds Emission-Line Survey (MCELS; Smith et al. 2005).

We are thus in a position to carefully examine the nebulae for this sample of SMC field OB stars that have known spectral types and radial velocities. In future work, we will systematically address the quantitative and varied properties of the entire field star sample, but the subset we present here provides especially compelling examples of objects that imply that the host stars were
Table 2

HiI Region Properties

\begin{tabular}{rlrccr}
\hline \hline Star & MCELS & $R(\operatorname{arcsec})$ & $F(\mathrm{H} \alpha)^{\mathrm{a}}$ & SB $^{\mathrm{b}}$ & $n_{e}\left(\mathrm{~cm}^{-3}\right)$ \\
\hline 1600 & S6 & 156.8 & $1.08 \mathrm{E}-11$ & $1.39 \mathrm{E}-16$ & 0.9 \\
3173 & S14 & 12.0 & $4.35 \mathrm{E}-12$ & $9.61 \mathrm{E}-15$ & 25.9 \\
17813 & S73 & 29.0 & $1.45 \mathrm{E}-12$ & $5.52 \mathrm{E}-16$ & 4.0 \\
24119 & S88 & 26.0 & $2.92 \mathrm{E}-12$ & $1.39 \mathrm{E}-15$ & 6.7 \\
35491 & $\ldots$ & 75.5 & $7.88 \mathrm{E}-12$ & $4.40 \mathrm{E}-16$ & 2.2 \\
36514 & S110 & 35.8 & $5.57 \mathrm{E}-12$ & $1.38 \mathrm{E}-15$ & 5.7 \\
64453 & S165 & 67.8 & $1.05 \mathrm{E}-12$ & $7.35 \mathrm{E}-17$ & 1.0 \\
66415 & S168 & 208.7 & $1.25 \mathrm{E}-11$ & $9.76 \mathrm{E}-17$ & 0.6 \\
67334 & $\ldots$ & 56.0 & $2.57 \mathrm{E}-12$ & $2.14 \mathrm{E}-14$ & 17.9 \\
69598 & S172 & 101.0 & $2.88 \mathrm{E}-12$ & $9.31 \mathrm{E}-17$ & 0.9 \\
70149 & S174 & 110.2 & $3.78 \mathrm{E}-12$ & $9.95 \mathrm{E}-17$ & 0.9 \\
71409 & S175 & 45.2 & $8.50 \mathrm{E}-13$ & $1.37 \mathrm{E}-16$ & 1.6 \\
71815 & S177 & 120.0 & $4.09 \mathrm{E}-12$ & $9.04 \mathrm{E}-17$ & 0.8 \\
75984 & S194 & 19.0 & $6.12 \mathrm{E}-13$ & $5.43 \mathrm{E}-16$ & 4.9 \\
\hline
\end{tabular}

${ }^{\mathrm{a}} \mathrm{erg} \mathrm{s}{ }^{-1} \mathrm{~cm}^{-2}$

b $\operatorname{erg~s}^{-1} \mathrm{~cm}^{-2} \operatorname{arcsec}^{-2}$

formed in place. We selected HiI regions that appear the most symmetric, with no evidence of bow shocks, and with target stars that are well centered within these nebulae. We examine these objects in both $\mathrm{H} \alpha$ and in the ratio map of $[\mathrm{OIII}] /[\mathrm{SII}]$, since the ionization structure confirms the general symmetry and allows us to identify objects with high optical depth (Pellegrini et al. 2012). In addition, we limit the sample to stars which show strong isolation with minimal evidence of companions.

Table 1 lists the sample. Columns 1, 2 and 3 respectively give the star's Massey (2002) identification, RIOTS4 spectral type from Lamb et al. (2013), and position from OGLE III (Udalski et al. 2008). Columns 4 and 5 list our RIOTS4 stellar radial velocity and the radial velocity of the nearest significant Hi component from Stanimirović et al. (1999), as described below. The OGLE $V$ magnitude and extinction $A_{V}$ from Zaritsky et al. (2002) are given in columns 6 and 7, respectively. Columns 8-12 list clustering parameters for each star described in $\S 3$ below.

Table 2 gives the properties of the HiI regions associated with the sample stars. Columns 1 and 2 list the star 
ID as before, and the nebular MCELS ID from Pellegrini et al. (2013). The nebular radius $R$, listed in column 3, is the mean of the nebular major and minor axes. The remaining columns give the $\mathrm{H} \alpha$ flux $F(\mathrm{H} \alpha), \mathrm{H} \alpha$ surface brightness (SB), and the nebular electron density $n_{e}$, derived from the emission measure implied by the SB and $R$. The surface brightnesses and electron densities of these objects in Table 2 are commensurate with those of typical HiI regions, and are an order of magnitude greater than the diffuse, warm, ionized component of the interstellar medium (ISM), where $n_{e}$ is on the order of $\sim 0.1 \mathrm{~cm}^{-3}$. We assume an SMC distance of $60 \mathrm{kpc}$ (e.g., Harries et al. 2003).

Figure 1 shows MCELS imaging of the sample, and Figure 2 shows OGLE $I$-band images. The nebular images in Figure 1 are constructed following Pellegrini et al. (2012). The left panel for each object shows $\mathrm{H} \alpha$, and the middle panel shows the $[\mathrm{SII}] /[\mathrm{OIII}]$ ratio image. The right panel shows the MCELS green continuum band with an effective wavelength of $5130 \AA$ and $\Delta \lambda=155 \AA$. Figure 1 shows that all of these field stars are significantly isolated and have symmetric nebulae that are difficult to explain if the stars are runaways ejected from clusters. The nebulae show regular, spherical morphology with a lower-ionization envelope traced by [SII] enclosing the high-ionization nebular center traced by [OIII]. This Strömgren-sphere ionization structure indicates that the nebulae are most likely optically thick (Pellegrini et al. 2012). Owing to their later spectral type in the early B-range, stars $17813,64453,67334$, and 75984 do not generate significant radiation in [OIII] and therefore do not show ionization stratification in Figure 1.

Figure[1]shows that the target stars are centered within these Strömgren spheres. The $\mathrm{H}$ recombination time $t_{r}=1 / n_{e} \alpha_{\mathrm{H}}$, where the recombination coefficient $\alpha_{\mathrm{H}}=$ $4 \times 10^{-13} \mathrm{~cm}^{3} \mathrm{~s}^{-1}$ for electron temperature $T_{e}=10^{4} \mathrm{~K}$. For $n_{e}=10 \mathrm{~cm}^{-3}, t_{r} \sim 10^{4} \mathrm{yr}$; a runaway star moving at $100 \mathrm{~km} \mathrm{~s}^{-1}$ will travel $\sim 1 \mathrm{pc}$ during this time. Thus, in such a dense medium, a runaway star will not noticeably "outrun" its nebula, and its Strömgren sphere will accompany its motion. However, in the diffuse ISM, $n_{e}$ is much lower, increasing $t_{r}$ proportionately. For a highdensity value of $n_{e}=1 \mathrm{~cm}^{-3}, t_{r} \sim 10^{5} \mathrm{yr}$, and a 100 $\mathrm{km} \mathrm{s}^{-1}$ runaway star will travel $10 \mathrm{pc}$. The Strömgren radius $R_{s} \sim 30 \mathrm{pc}$ for an $09.5 \mathrm{~V}$ star, and so in this case, the star will appear significantly off-center, in a nebula that is elongated along the direction of motion. Stars traveling with such high speeds should also show bow shocks. If the star is within the diffuse, ionized medium, then $n_{e}$ is even lower, on the order of $0.1 \mathrm{~cm}^{-3}$, with an even longer-lived nebula.

Furthermore, runaway stars with velocities strongly dominated by the line-of-sight component will remain close to their parent cluster in projection. As shown in Figure 2, this is not seen for our sample objects. In any case, for this sample of stars, the radial velocities (RV) shown in Table 1 are selected to be within 10 $\mathrm{km} \mathrm{s}^{-1}$ of the nearest Hi components having brightness temperature $\geq 20 \mathrm{~K}$, as mapped by Stanimirović et al. (1999). This is a nominal criterion for the presence of significant $\mathrm{HI}$ in the line of sight used by Lamb et al. (2013b, in preparation). Figure 3 shows that the stellar RVs show excellent correspondence with the HI velocity components, consistent with the scenario that these stars formed near their currently observed locations. Four objects, 1600, 17813, 69598, and 70149, fall outside the Hi threshold criterion and might be candidate runaway stars. However, as we shall see below, other criteria suggest that most of these are also in-situ field stars.

We also note that some nebulae, in particular those for 66415 and 69598 , show morphology near their periphery suggesting photo-evaporation by the stellar UV radiation ( $\mathrm{H} \alpha$ images in Figure 11). Such features are common in star-forming regions, and in extreme form correspond to "elephant trunk" structures pointing radially at the star. The presence of large-scale photo-evaporated morphology again strongly suggests that the surrounding nebulae are indeed the natal clouds for the enclosed O stars.

\section{DEGREE OF ISOLATION}

We investigate the degree of isolation for these stars more quantitatively using data from the OGLE-III survey (Udalski et al. 2008) and a friends-of-friends algorithm (Battinelli 1991; Lamb et al. 2010). We first determine the clustering length in a $18^{\prime} \times 9^{\prime}$ field within which the target star is found, using all stars brighter than a completeness limit of $20.5 \mathrm{mag}$ in $I$. The clustering length $l$ is the projected distance that maximizes the number of clusters consisting of 3 or more stars found within $l$ of a cluster member. Most of the fields show $l \sim 1-2 \mathrm{pc}$, depending on mean stellar density. Since we are only interested in companion stars that are physically associated with the target, we distinguish between main sequence stars, which we assume to be associated, and other stars, which we assume to be background or foreground stars. We thus apply a conservative color criterion of $V-I<0.5$, which discriminates between main sequence stars and Galactic contamination, which becomes significant at $V-I>0.5$, according to the Besançon stellar population synthesis models for the Galaxy (Robin et al. 2003). Table 1 gives $l$ for each target star, the total number of companion stars $N$ (tot) identified by the friends-of-friends algorithm, and the number of companions $N(\mathrm{MS})$ considered to be main sequence stars, and therefore physically associated with the target $\mathrm{OB}$ star. We list the identified companion stars themselves, with their photometry, in Table 3. The last column of Table 3 indicates which of these companions are identified as main sequence stars.

More than one-third of the field OB stars (5 out of 14) show no identified cluster at the completeness depth of the survey field. This is a conservative frequency, since the friends-of-friends clustering identification includes contaminating background stars. Furthermore, small-number statistics will also introduce a few spurious cluster detections. As noted above, four stars have RVs falling outside those of the line-of-sight HI components. Of these, 1600 and 69598 show 2 and 6 associated companion stars, respectively (Table 1). It is therefore extremely likely that 69598 formed in situ with this sparse group. The other two stars, 17813 and 70149, show no evidence of companion stars.

We follow the sparse cluster analysis by Lamb et al. (2010), examining the ratio between the mass $m_{2}$ of the second-highest mass star to that of the target star $m_{1}$ for each identified group of companions. The target masses are from Lamb et al. (2013), and are simply based on 


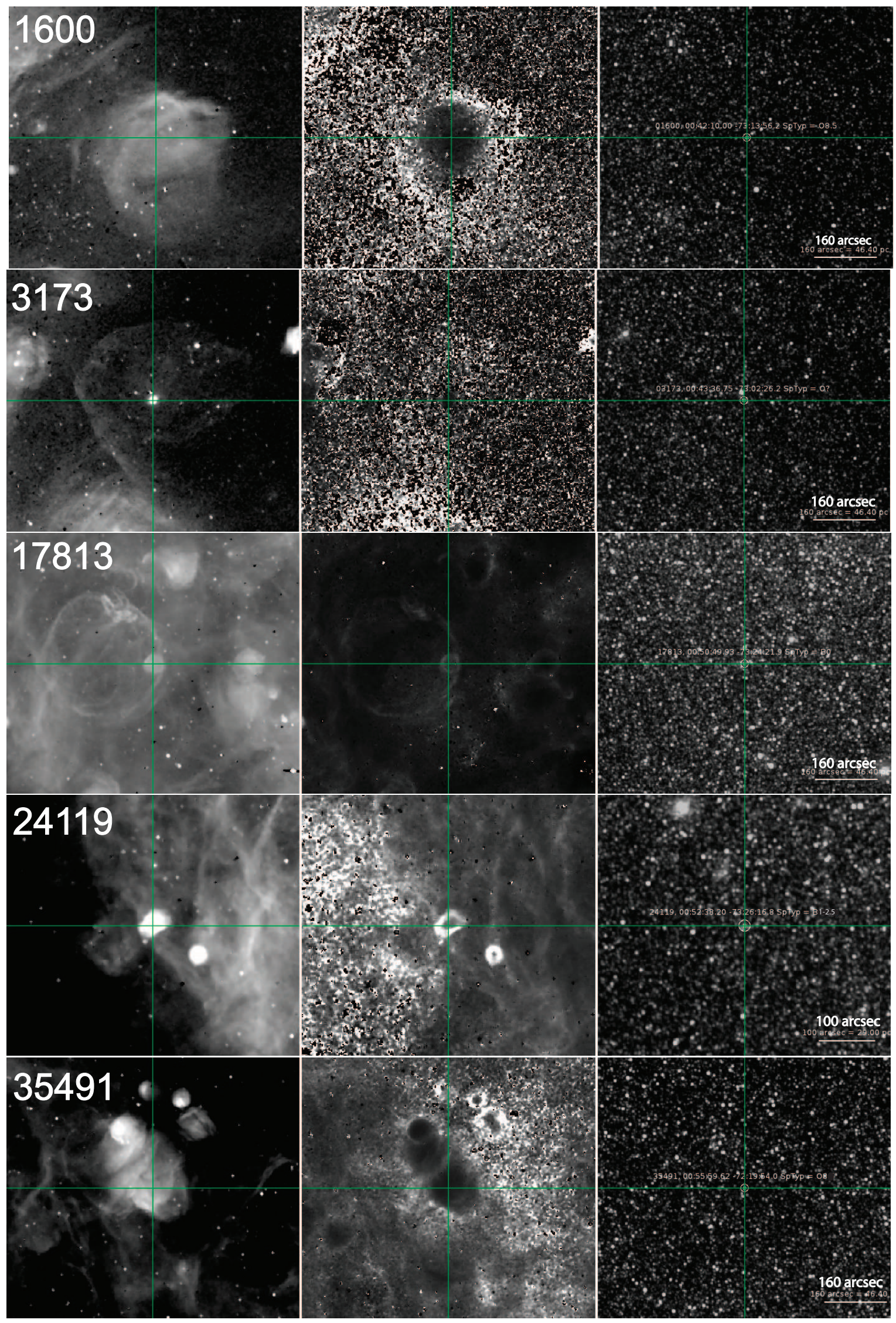

Figure 1. Images in (left to right) $\mathrm{H} \alpha$, [SII]/[OIII], and $\lambda 5130$ continuum, with the cross hairs centered on the target stars. Strong [SII] is black in the center panels. The $160^{\prime \prime}$ and $100^{\prime \prime}$ scale bars correpond to $46.4 \mathrm{pc}$ and $29.0 \mathrm{pc}$ at the SMC distance, respectively. N is up and $\mathrm{E}$ to the left. 


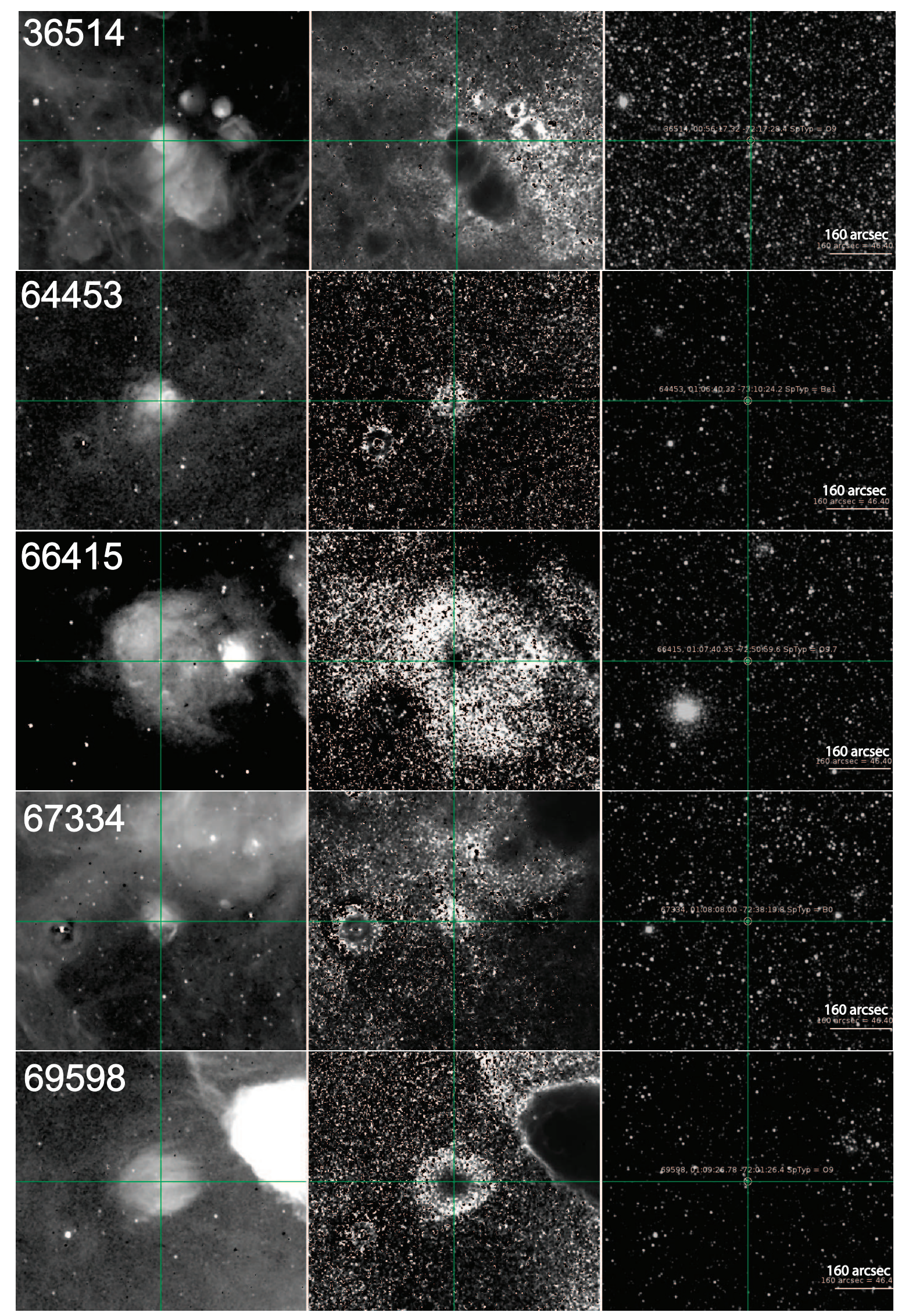

Figure 1. - continued. 


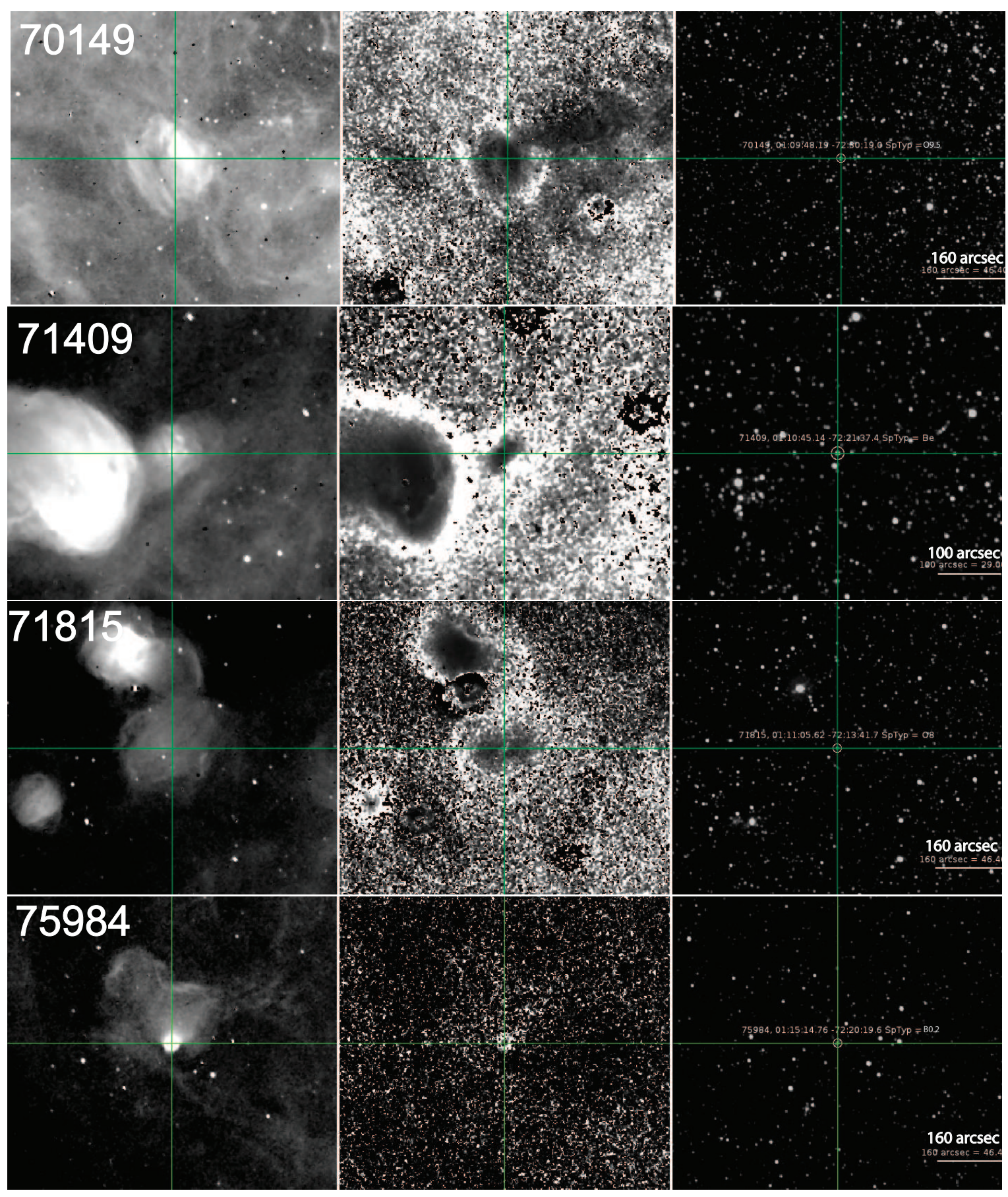

Figure 1. - continued.

their photometry and spectral types. We obtain masses for the companion stars using the statistical method from Lamb et al. (2013), using OGLE III photometry. This is done by generating a probability distribution for the observed VI photometry based on the photometric error and the measured extinction from Zaritsky et al. (2002). The extinction $A_{V}$ for each star is obtained by averaging $A_{V}$ for all stars within a 1 arcmin radius, and the adopted uncertainty is the standard deviation of these extinction values. For each companion star, we generate $10^{4}$ independent realizations of its $V$ magnitude, $I$ magnitude, and extinction by selecting a random value from a Gaussian distribution centered on their measured val- ues. These realizations are compared with evolutionary tracks at SMC metallicity from Charbonnel et al. (1993) to obtain a stellar mass for each realization. Any realizations existing outside the model parameter space are discarded as unphysical. The remaining realizations are averaged to obtain our adopted mass for each companion star. Further details on this technique can be found in Lamb et al. (2013). Table 1 lists the target star masses and the mass ratios $m_{2} / m_{1}$, while Table 3 gives mass estimates for all the identified companion stars.

Table 3 demonstrates that most of the target field OB stars do show evidence of companion stars. If these are indeed physically associated with the targets, it provides 
Table 3

Companion stars

\begin{tabular}{|c|c|c|c|c|c|c|c|c|}
\hline \multicolumn{2}{|c|}{$\mathrm{RA}$ and $\operatorname{Dec}(\mathrm{J} 2000)^{\mathrm{a}}$} & $V^{\mathrm{a}}$ & $I^{\mathrm{a}}$ & $V$ err $^{\mathrm{a}}$ & $I \mathrm{err}^{\mathrm{a}}$ & $A_{V}{ }^{\mathrm{b}}$ & $m\left(\mathrm{M}_{\odot}\right)$ & $\mathrm{MS}^{\mathrm{c}}$ \\
\hline \multicolumn{9}{|c|}{1600} \\
\hline $0: 42: 10.43$ & $-73: 13: 58.2$ & 19.786 & 19.855 & 0.119 & 0.200 & 0.43 & 2.3 & $\mathrm{Y}$ \\
\hline 0:42:09.44 & $-73: 13: 57.9$ & 19.821 & 18.850 & 0.098 & 0.081 & 0.41 & 1.9 & $\mathrm{~N}$ \\
\hline 0:42:09.62 & $-73: 13: 55.9$ & 19.075 & 18.535 & 0.128 & 0.102 & 0.38 & 2.5 & $\mathrm{Y}$ \\
\hline \multicolumn{9}{|c|}{3173} \\
\hline $0: 43: 36.83$ & $-73: 02: 28.9$ & 18.197 & 18.824 & 0.094 & 0.112 & 0.36 & 4.7 & $\mathrm{Y}$ \\
\hline $0: 43: 36.97$ & $-73: 02: 28.3$ & $\ldots$ & 18.949 & $\ldots$ & 0.130 & 0.38 & $\cdots$ & $\cdots$ \\
\hline $0: 43: 37.28$ & $-73: 02: 25.5$ & 19.976 & 19.514 & 0.248 & 0.177 & 0.37 & 2.0 & $\mathrm{Y}$ \\
\hline $0: 43: 36.28$ & $-73: 02: 28.4$ & 18.438 & 19.119 & 0.103 & 0.129 & 0.37 & 4.1 & $\mathrm{Y}$ \\
\hline 0:43:36.53 & $-73: 02: 28.0$ & 17.686 & 18.611 & 0.065 & 0.106 & 0.37 & $\cdots$ & $\cdots$ \\
\hline \multicolumn{9}{|c|}{24119} \\
\hline $0: 52: 38.30$ & $-73: 26: 15.2$ & 19.372 & 19.342 & 0.325 & 0.190 & 0.25 & 2.4 & $\mathrm{Y}$ \\
\hline 0:52:37.39 & $-73: 26: 17.7$ & 20.138 & 19.827 & 0.115 & 0.203 & 0.26 & 1.9 & $\mathrm{Y}$ \\
\hline 0:52:37.66 & $-73: 26: 18.4$ & 19.847 & 19.763 & 0.097 & 0.175 & 0.26 & 2.2 & $\mathrm{Y}$ \\
\hline 0:52:37.92 & $-73: 26: 14.4$ & 19.609 & 18.843 & 0.085 & 0.074 & 0.26 & 2.3 & $\mathrm{~N}$ \\
\hline \multicolumn{9}{|c|}{36514} \\
\hline $0: 56: 17.80$ & $-72: 17: 30.1$ & 20.561 & 20.504 & 0.157 & 0.316 & 0.32 & 1.8 & $\mathrm{Y}$ \\
\hline 0:56:17.99 & $-72: 17: 27.2$ & 20.881 & 20.953 & 0.194 & 0.427 & 0.32 & 1.7 & $\mathrm{Y}$ \\
\hline $0: 56: 18.25$ & $-72: 17: 31.4$ & $\ldots$ & 21.096 & $\ldots$ & 0.363 & 0.33 & $\cdots$ & $\cdots$ \\
\hline $0: 56: 18.28$ & $-72: 17: 24.0$ & 19.835 & 18.801 & 0.060 & 0.060 & 0.33 & 1.7 & $\mathrm{~N}$ \\
\hline $0: 56: 18.59$ & $-72: 17: 28.7$ & 21.298 & 21.121 & 0.186 & 0.370 & 0.33 & 1.5 & $\mathrm{Y}$ \\
\hline $0: 56: 18.69$ & $-72: 17: 26.0$ & 19.642 & 18.656 & 0.063 & 0.056 & 0.33 & 1.8 & $\mathrm{~N}$ \\
\hline $0: 56: 18.75$ & $-72: 17: 33.1$ & 19.560 & 19.556 & 0.048 & 0.121 & 0.35 & 2.5 & $\mathrm{Y}$ \\
\hline $0: 56: 18.96$ & $-72: 17: 33.6$ & 19.987 & 19.566 & 0.068 & 0.126 & 0.35 & 2.0 & $\mathrm{Y}$ \\
\hline $0: 56: 18.98$ & $-72: 17: 28.0$ & 20.402 & 20.198 & 0.108 & 0.195 & 0.32 & 1.9 & $\mathrm{Y}$ \\
\hline 0:56:19.43 & $-72: 17: 26.5$ & 21.330 & 20.687 & 0.214 & 0.279 & 0.33 & 1.5 & $\mathrm{Y}$ \\
\hline 0:56:16.30 & $-72: 17: 27.2$ & 19.528 & 18.752 & 0.056 & 0.056 & 0.34 & 2.3 & $\mathrm{~N}$ \\
\hline $0: 56: 16.88$ & $-72: 17: 28.3$ & $\cdots$ & 19.022 & $\cdots$ & 0.085 & 0.33 & $\cdots$ & $\cdots$ \\
\hline \multicolumn{9}{|c|}{64453} \\
\hline 1:06:40.96 & $-73: 10: 25.9$ & 19.611 & 19.228 & 0.646 & 0.375 & 0.29 & 2.2 & $\mathrm{Y}$ \\
\hline 1:06:41.15 & $-73: 10: 27.6$ & 20.057 & 20.235 & 0.218 & 0.271 & 0.31 & 2.1 & $\mathrm{Y}$ \\
\hline \multicolumn{9}{|c|}{66415} \\
\hline 1:07:41.13 & $-72: 51: 04.0$ & 19.504 & 18.746 & 0.077 & 0.065 & 0.25 & 2.4 & $\mathrm{~N}$ \\
\hline 1:07:41.12 & $-72: 50: 51.6$ & 20.128 & 20.207 & 0.073 & 0.182 & 0.26 & 2.2 & $\mathrm{Y}$ \\
\hline 1:07:41.47 & $-72: 50: 59.4$ & 18.653 & 18.002 & 0.056 & 0.034 & 0.26 & 3.0 & $\mathrm{~N}$ \\
\hline 1:07:41.45 & $-72: 50: 55.4$ & 19.253 & 19.331 & 0.037 & 0.098 & 0.27 & 2.9 & $\mathrm{Y}$ \\
\hline \multicolumn{9}{|c|}{67334} \\
\hline 1:08:08.79 & $-72: 38: 21.1$ & 17.668 & 16.396 & 0.019 & 0.010 & 0.41 & 2.4 & $\mathrm{~N}$ \\
\hline 1:08:07.05 & $-72: 38: 16.1$ & 20.026 & 19.069 & 0.063 & 0.075 & 0.41 & 1.9 & $\mathrm{~N}$ \\
\hline 1:08:07.33 & $-72: 38: 16.6$ & 18.663 & 17.537 & 0.032 & 0.019 & 0.42 & 2.0 & $\mathrm{~N}$ \\
\hline 1:08:07.90 & $-72: 38: 23.5$ & 18.806 & 18.835 & 0.032 & 0.065 & 0.40 & 3.1 & $\mathrm{Y}$ \\
\hline \multicolumn{9}{|c|}{69598} \\
\hline 1:09:27.27 & $-72: 01: 21.3$ & 20.841 & 19.955 & 0.154 & 0.151 & 0.22 & 1.6 & $\mathrm{~N}$ \\
\hline 1:09:27.48 & $-72: 01: 23.2$ & 17.497 & 17.673 & 0.013 & 0.023 & 0.22 & 5.7 & $\mathrm{Y}$ \\
\hline 1:09:27.61 & $-72: 01: 25.4$ & 17.919 & 18.105 & 0.014 & 0.033 & 0.22 & 5.1 & $\mathrm{Y}$ \\
\hline 1:09:28.10 & $-72: 01: 30.6$ & 18.639 & 18.724 & 0.023 & 0.055 & 0.21 & 3.6 & $\mathrm{Y}$ \\
\hline 1:09:28.11 & $-72: 01: 40.9$ & 16.094 & 16.283 & 0.008 & 0.010 & 0.23 & 8.9 & $\mathrm{Y}$ \\
\hline 1:09:28.26 & $-72: 01: 20.3$ & 16.728 & 15.741 & 0.009 & 0.008 & 0.20 & 4.9 & $\mathrm{~N}$ \\
\hline 1:09:28.44 & $-72: 01: 26.5$ & 20.750 & 19.859 & 0.125 & 0.147 & 0.19 & 1.6 & $\mathrm{~N}$ \\
\hline 1:09:28.82 & $-72: 01: 39.8$ & 20.453 & 19.684 & 0.092 & 0.117 & 0.22 & 1.8 & $\mathrm{~N}$ \\
\hline 1:09:28.99 & $-72: 01: 33.9$ & 18.617 & 18.712 & 0.018 & 0.056 & 0.22 & 3.7 & $\mathrm{Y}$ \\
\hline 1:09:29.19 & $-72: 01: 43.3$ & 20.620 & 19.637 & 0.146 & 0.112 & 0.22 & 1.6 & $\mathrm{~N}$ \\
\hline 1:09:29.54 & $-72: 01: 33.4$ & 19.143 & 19.242 & 0.036 & 0.086 & 0.20 & 3.2 & $\mathrm{Y}$ \\
\hline
\end{tabular}




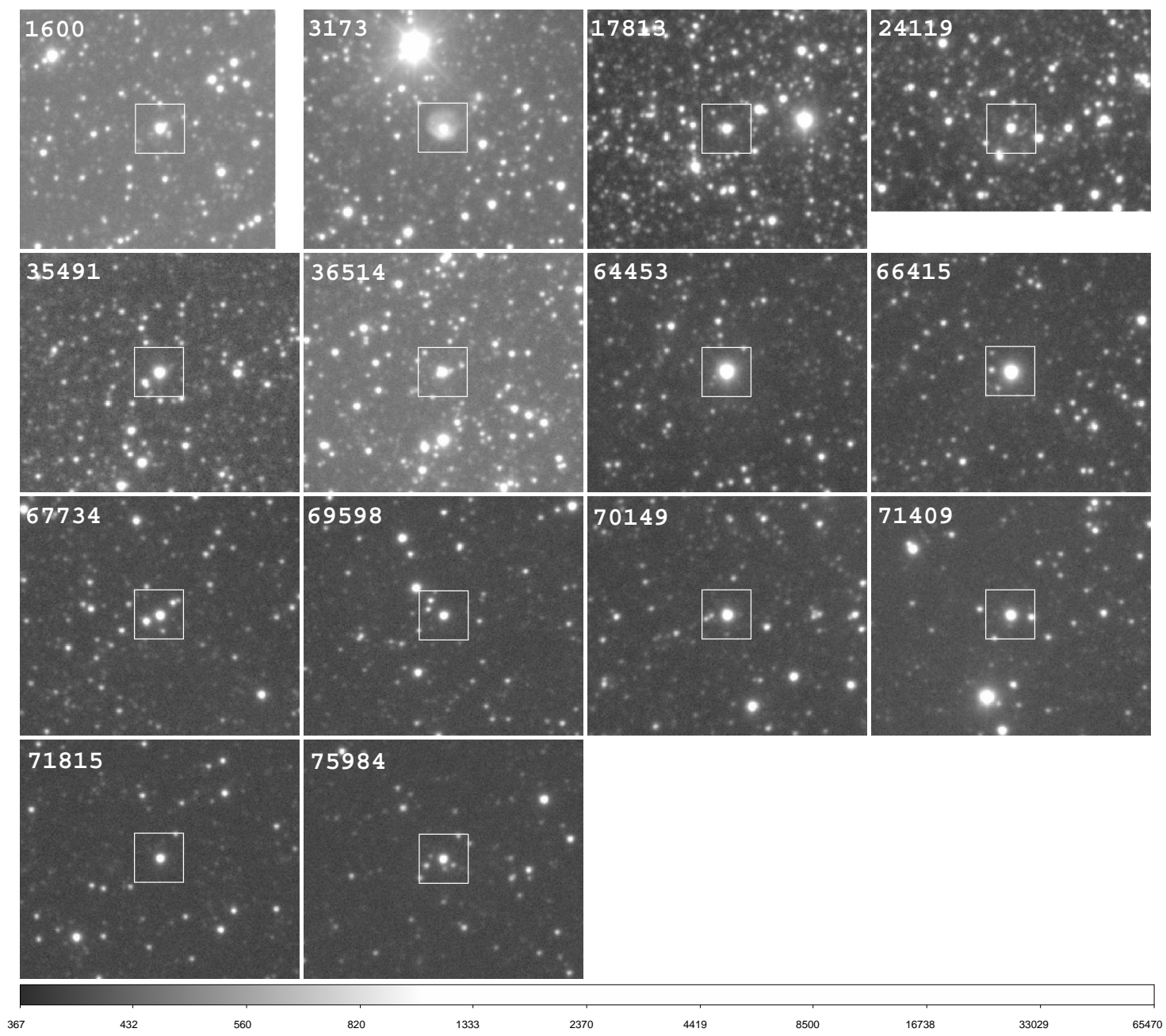

Figure 2. OGLE $I$-band images of the sample objects. The target stars are centered within $12^{\prime \prime}$ boxes (3.4 pc). N is up, E to the left.

Table 3

Companion stars (continued)

\begin{tabular}{|c|c|c|c|c|c|c|c|c|}
\hline \multicolumn{2}{|c|}{$\mathrm{RA}$ and Dec $(\mathrm{J} 2000)^{\mathrm{a}}$} & $V^{\mathrm{a}}$ & $I^{\mathrm{a}}$ & $V$ err $^{\mathrm{a}}$ & $I \mathrm{err}^{\mathrm{a}}$ & $A_{V}{ }^{\mathrm{b}}$ & $m\left(\mathrm{M}_{\odot}\right)$ & $\mathrm{MS}^{\mathrm{c}}$ \\
\hline \multicolumn{9}{|c|}{70149} \\
\hline$\overline{1: 09: 49.13}$ & $-72: 30: 19.5$ & 19.701 & 18.789 & 0.074 & 0.066 & 0.31 & 2.0 & $\mathrm{~N}$ \\
\hline $1: 09: 49.40$ & $-72: 30: 20.3$ & 19.400 & 18.506 & 0.048 & 0.048 & 0.32 & 2.1 & $\mathrm{~N}$ \\
\hline $1: 09: 48.27$ & $-72: 30: 24.0$ & $\cdots$ & 20.349 & $\cdots$ & 0.214 & 0.31 & $\cdots$ & $\cdots$ \\
\hline \multicolumn{9}{|c|}{75984} \\
\hline $1: 15: 15.37$ & $-72: 20: 18.7$ & 20.238 & 19.677 & 0.122 & 0.140 & 0.30 & 1.8 & $\mathrm{Y}$ \\
\hline $1: 15: 15.61$ & $-72: 20: 21.0$ & 17.992 & 18.155 & 0.023 & 0.036 & 0.30 & 5.0 & $\mathrm{Y}$ \\
\hline $1: 15: 15.95$ & $-72: 20: 24.0$ & 18.501 & 18.640 & 0.024 & 0.050 & 0.36 & 4.0 & $\mathrm{Y}$ \\
\hline $1: 15: 13.26$ & $-72: 20: 15.7$ & 19.918 & 18.972 & 0.052 & 0.067 & 0.32 & 1.8 & $\mathrm{~N}$ \\
\hline $1: 15: 13.62$ & $-72: 20: 36.2$ & 19.130 & 18.189 & 0.035 & 0.035 & 0.37 & 2.3 & $\mathrm{~N}$ \\
\hline $1: 15: 13.88$ & $-72: 20: 35.2$ & 21.018 & 20.059 & 0.172 & 0.170 & 0.37 & 1.5 & $\mathrm{~N}$ \\
\hline $1: 15: 13.87$ & $-72: 20: 13.8$ & 18.335 & 18.494 & 0.024 & 0.045 & 0.29 & 4.5 & $\mathrm{Y}$ \\
\hline $1: 15: 14.04$ & $-72: 20: 07.8$ & 20.413 & 19.475 & 0.093 & 0.109 & 0.30 & 1.6 & $\mathrm{~N}$ \\
\hline $1: 15: 14.18$ & $-72: 20: 20.9$ & 18.662 & 18.802 & 0.026 & 0.064 & 0.34 & 3.9 & $\mathrm{Y}$ \\
\hline $1: 15: 14.63$ & $-72: 20: 21.7$ & 19.613 & 19.369 & 0.075 & 0.104 & 0.31 & 2.1 & $\mathrm{Y}$ \\
\hline $1: 15: 14.71$ & $-72: 20: 29.8$ & 19.398 & 19.486 & 0.048 & 0.112 & 0.40 & 2.8 & $\mathrm{Y}$ \\
\hline
\end{tabular}

${ }^{a}$ From OGLE III (Udalski et al. 2008).

b From Zaritsky et al. (2002).

${ }^{c}$ Indicates whether star is considered to be on the main sequence. 

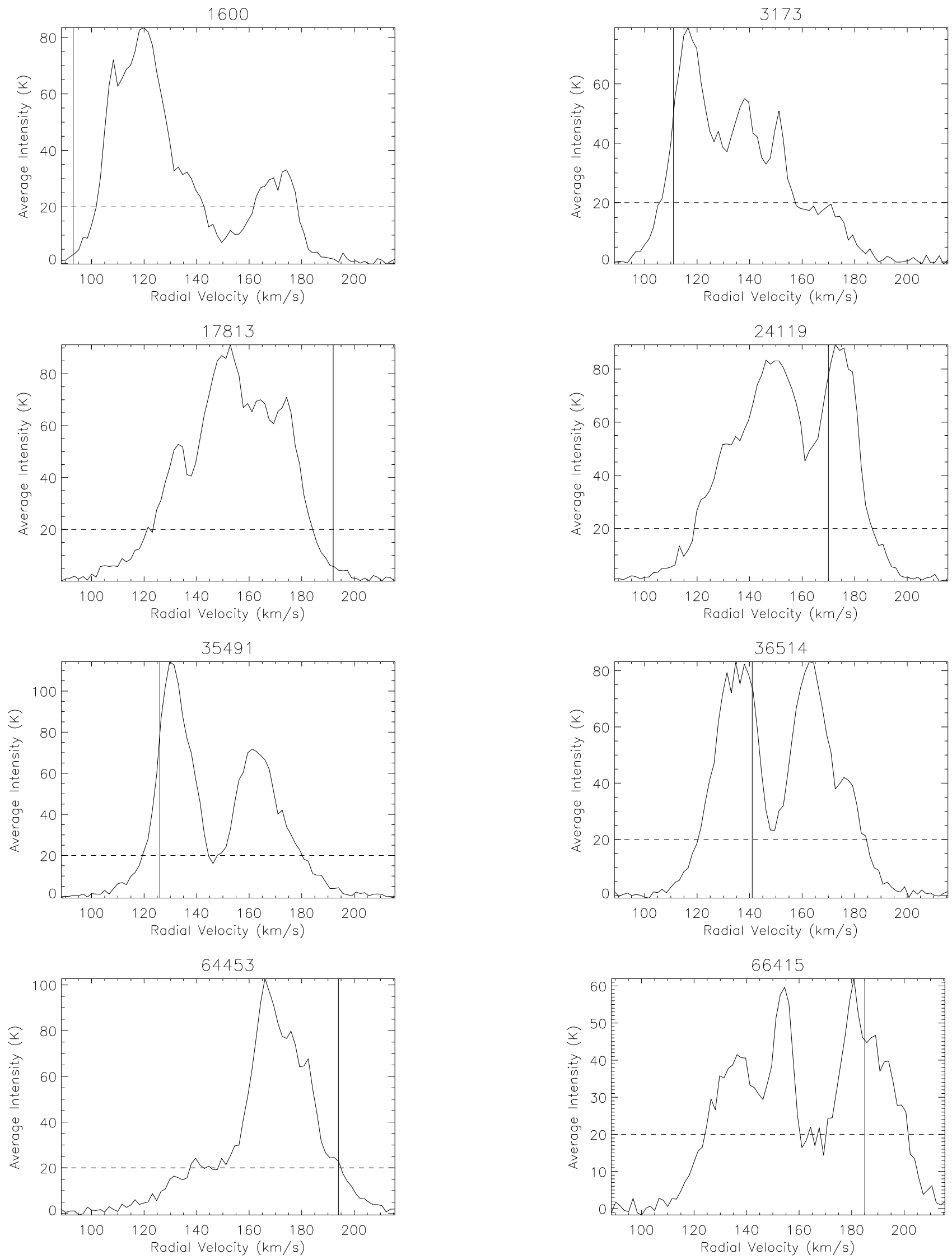

Figure 3. The Hi velocity structure within a $1^{\prime} \times 1^{\prime}$ aperture centered on each target star from the Hi survey by Stanimirović et al. (1999), plotted as brightness temperature vs radial velocity. The solid vertical line shows the star's measured RV, and the dashed lines shows a nominal criterion for significant Hi (see text). For star 75984 , note that Hi is only observed for velocities $<215 \mathrm{~km} \mathrm{~s}{ }^{-1}$, and so the abrupt drop-off at the high-velocity end is likely an artifact. 

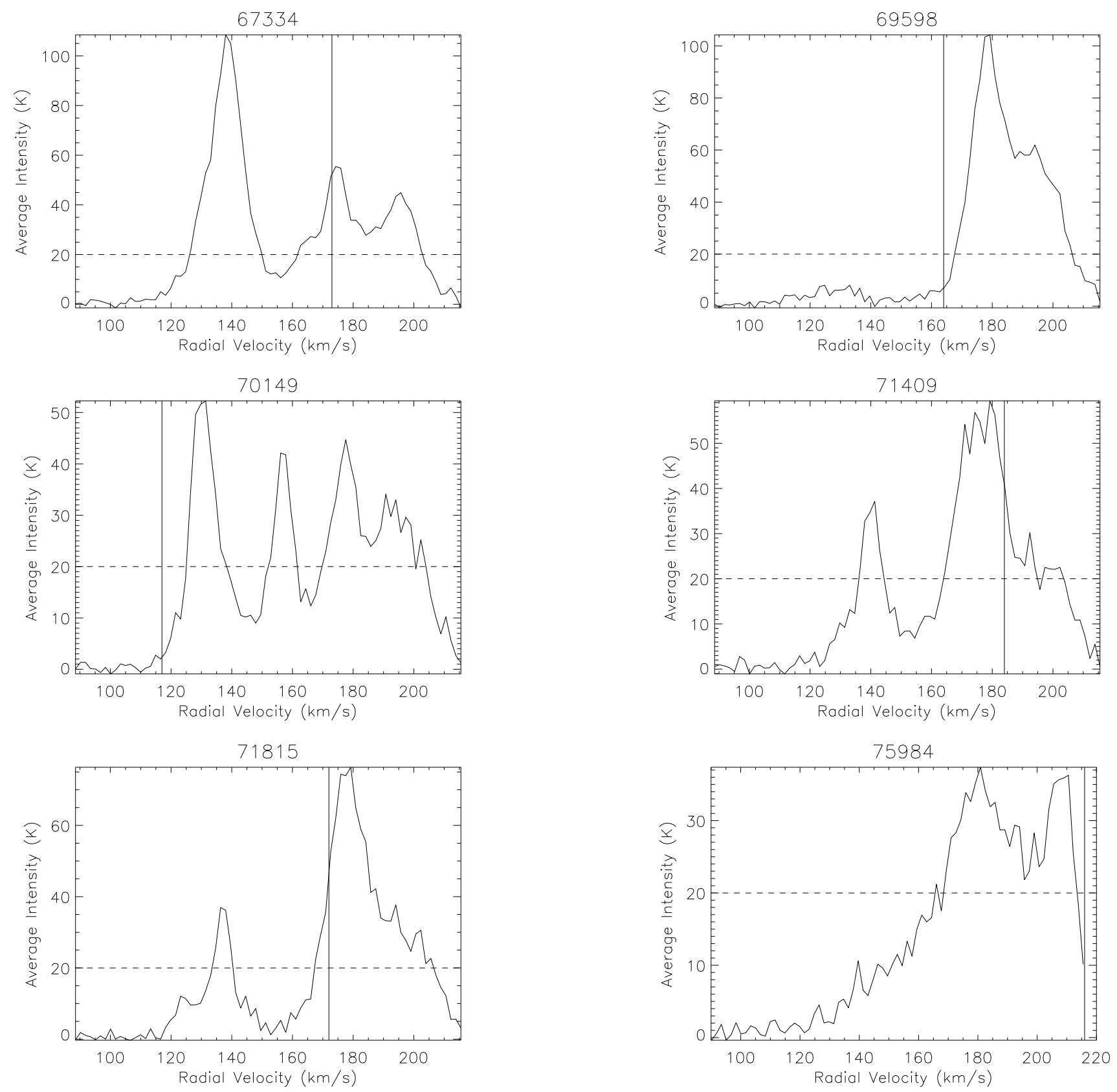

Figure 3. - continued. 
even stronger evidence that these OB stars formed in situ. The mass ratios $m_{2} / m_{1}$ may be slightly larger than those reported by Lamb et al. (2010), whose maximum $m_{2} / m_{1}$ is 0.17 . However, that work was based on much deeper observations with the Hubble Space Telescope, for a smaller sample; whereas it is likely that some of our OGLE stars are in fact unresolved multiples. We also note our high frequency of non-detections of companions. As discussed by Lamb et al. (2010), while their $m_{2} / m_{1}$ ratios are low, they are still expected with reasonable probabilities of $10-20 \%$ if the IMF and cluster mass functions are independent. This contrasts with scenarios in which the maximum-mass star depends on cluster mass (e.g., Weidner \& Kroupa 2006; Vanbeveren 1982).

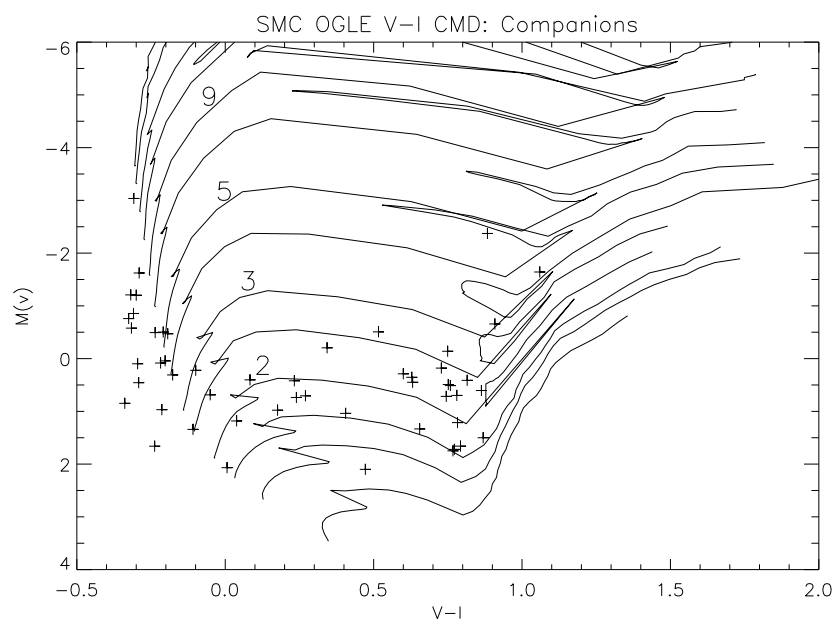

Figure 4. Composite color-magnitude diagram of all the stars identified to be in sparse groups in Table 3, excluding the target OB stars. Evolutionary tracks from Charbonnel et al. (1993) are overlaid, with model stellar masses shown.

Figure 4 shows the color-magnitude diagram for all the identified companion stars in Table 3. We generate a composite IMF for all the identified clusters, following the formalism of Scalo (1986) and assuming a single, power-law form, having slope $\Gamma$ given by,

$$
\Gamma=d \log \xi(\log m) / d \log m,
$$

where $\xi(\log m)$ is the mass function given in units of stars born per logarithmic stellar mass $m\left(M_{\odot}\right)$ per unit area $\left(\mathrm{kpc}^{2}\right)$. We obtain $\xi(\log m)$ by counting the number of stars per mass bin and dividing by the area covered by the observations. We account for the different size mass bins by normalizing each bin to one dex in mass. When constructing the IMF, our star counts include only the companion stars identified as main sequence stars above (Table 3).

Figure 5 shows the composite mass function for all the target stars with companions. Since the sample selection criteria are based on field OB star status, the composite IMF must be much flatter than the usual Salpeter powerlaw slope of $\Gamma=-1.35$. Figure 5 shows that this is indeed the case, with the composite IMF slope of $\Gamma=$ $-0.4 \pm 0.6$. This is similar to the composite slope for companions found by Lamb et al. (2010) of $\Gamma=0.1 \pm 1.0$. Excluding the target stars, the composite IMF slope is $\Gamma=-1.6 \pm 1.0$, which turns out to be consistent with the Salpeter value (Figure 6).
Finally, we note that five of the 14 target stars, 17813, 35491, 70149, 71409, and 71815, show no evidence of associated companions down to the completeness limit of $I=20.5$ (Table 1). We further examined these fields for detected stars fainter than the completeness limit, and only one target, 17813, shows an additional detected companion fainter than this threshold. If these five OB stars formed in situ, as is suggested by our fairly rigorous selection criteria, then they set even stronger limits to the star formation density than the rest of the sample. This includes the possibility that they formed in complete isolation. The stars 17813 and 70149 are also among the objects whose RV falls outside the observed Hi kinematic components (Figure 3), and thus these objects have the highest probability of being runaway stars. However, the difference in velocities is only by 8 and $9 \mathrm{~km} \mathrm{~s}^{-1}$, respectively, and as argued earlier, the spherical symmetry of the nebulae, MCELS-S73 and MCELS-S174, is hard to explain if the stars are runaways (Figure 1). The nebular densities are also fully commensurate with the normal densities of star-forming HiI regions (Table 2).

We have not addressed the possibility that the target field $\mathrm{OB}$ stars are binaries. Given that the majority of OB stars in clusters are binaries (e.g., Sana et al. 2012), it is reasonable to expect that many, if not most of the field OB stars are binaries as well. To date, none of the stars in our sample are known binary stars. Whether or not any turn out to be binaries will also offer vital diagnostics of star formation models, for example, whether the binary frequency is different among field OB stars relative to that in clusters.

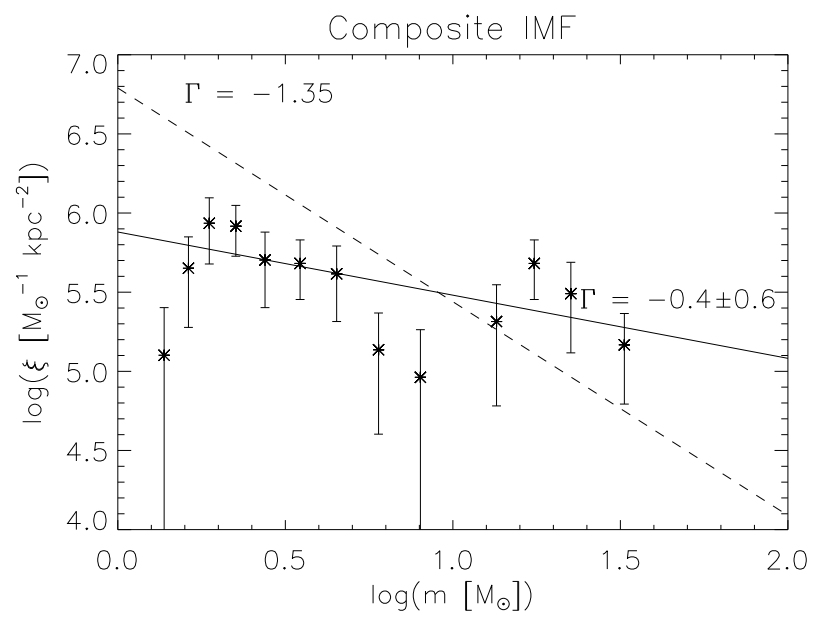

Figure 5. Composite IMF of all the sparse groups in Table 3 including the target OB stars. The fitted slope is shown with a solid line, while the dashed line shows the Salpeter value. Root- $N$ error bars are shown.

\section{CONCLUSION}

In summary, we present a sample of 14 isolated OB stars that appear to have formed in the field, under extremely sparse star-forming conditions. The stars are selected to be at least $28 \mathrm{pc}$ from any other OB star, and they are centered within fairly circular, symmetric HII regions, with no evidence of bow shocks, implying that they are not runaway stars with transverse velocities. They are also not line-of-sight runaways, based on their radial 


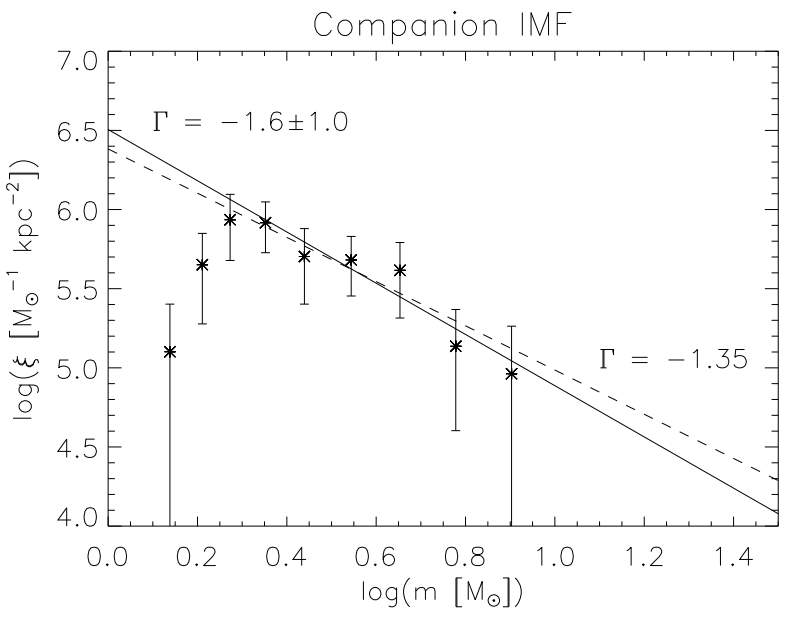

Figure 6. Same as Figure 5 but excluding the target OB stars.

velocities relative to local $\mathrm{HI}$, and the fact that no obvious parent clusters are near the line of sight. HiI regions generated by two of the stars show evidence of photoevaporated structures, as seen in other star-forming regions. We carried out a friends-of-friends analysis of the stellar fields for our sample, which shows that most of these OB stars have $\lesssim 7$ main sequence companions in projection. The presence of companions, if they are indeed physically associated, further implies that the target stars formed in situ. However, more than one third of the sample (5 out of 14 stars) shows no companions above the completeness limit, and it remains possible that these OB stars formed in complete isolation.

Our sample of stars presents some of the strongest evidence to date that massive OB stars can and do form in relative isolation. Their existence poses strong empirical constraints on theories of star formation and challenges proposed relations between cluster mass and maximum stellar mass. We also stress that while this sample represents strong selection criteria, there are many more objects that may similarly have formed in situ.

This work was supported by NSF grants AST-0907758 and AST-0806476. C.T.K. was supported by the Undergraduate Research Opportunities Program at the University of Michigan. We thank Anne Jaskot for assistance with the MCELS data.

\section{REFERENCES}

Battinelli, P. 1991, A\&A, 244, 69

Bressert, E., et al. 2012, A\&A 542, A49

Burki, G. 1978, A\&A 62, 159

Elmegreen, B. G. \& Efremov, Y. N. 1997, ApJ, 480, 235

de Wit, W. J., Testi, L., Palla, F., Vanzi, L., \& Zinnecker, H. 2004, A\&A 425, 937

de Wit, W. J., Testi, L., Palla, F., \& Zinnecker, H. 2005, A\&A 437, 247

Gies, D. R. 1987, ApJS 64, 545

Guthrie, B. N. G. 1984, MNRAS, 210, 159

Gvaramadze, V. V., Weidner, C., Kroupa, P., \& Pflamm-Altenburg, J. MNRAS 424, 3037

Huang, W. \& Gies, D. R. 2008, ApJ 683, 1045

Lamb, J. B., Oey, M. S., Graus, A. S., Adams, F. C., \& Segura-Cox, D. M. 2013, ApJ, 763, 101

Lamb, J. B., Oey, M. S., Werk, J. K., \& Ingleby, L. D. 2010, ApJ 725,1886

Massey, P. 2002, ApJS 141, 81

Massey, P., Johnson, K. E., \& Degioia-Eastwood, K. 1995, ApJ 454,151

Moffat, A. F. J., et al. 1998, A\&A 331, 949

Oey, M. S. 2011, ApJL, 739, L46

Oey, M. S. \& Clarke, C. J. 1998, AJ 115, 1543

Oey, M. S. \& Lamb, J. B. 2012, in Four Decades of Research on Massive Stars, eds. L. Drissen, C. Robert, \& N. St-Louis, (San Francisco: ASP), 431

Oey, M. S., King, N. L., \& Parker, J. W. 2004, AJ 127, 1632

Pellegrini, E. W., Oey, M. S., Winkler, P. F., Points, S. D., \& Smith, R. C. 2012, ApJ 755, 40; Erratum in press, astro-ph/1202.3334

Robin, A. C., Reylé, C., Derrière, S., \& Picaud, S. 2003, A\&A 409, 523

Sana, H., et al. 2012, Science, 337, 444

Scalo, J. M. 1986, Fund. Cosmic Physics, 11, 1

Selier, R., Heydari-Malayeri, M., \& Gouliermis, D. A. 2011, A\&A 529,40

Salpeter, E. E. 1955, ApJ 121, 161

Smith, R. C., et al. 2005, BAAS 37, 1200

Stanimirović, S., Staveley-Smith, L., Dickey, J. M., Sault, R. J., \& Snowden, S. L., MNRAS 302, 417

Testi, L., Palla, F., Prusti, T., Natta, A., \& Maltagliati, S. 1997, A\&A 320,159

Testi, L., Palla, F. \& Natta, A. 1999, A\&A 342, 515

Udalski, A., et al. 2008, AcA 58, 329

Vanbeveren, D. 1982, A\&A 115, 65

Weidner, C. \& Kroupa, P. 2006, MNRAS 365, 1333

Wolff, S. C., Strom, S. E., Dror, D. \& Venn, K. 2007, AJ 133, 1092

Zaritsky, D., Harris, J., Thompson, I. B., Grebel, E. K., \& Massey, P. 2002, AJ 123, 855

Zastrow, J., Oey, M. S., \& Pellegrini, E. W. 2013, ApJ, submitted; astro-ph/1212.5487 